\title{
Spatial Coherence Properties of One Dimensional Exciton-Polariton Condensates
}

\author{
J. Fischer, ${ }^{1}$ I. G. Savenko, ${ }^{2,3}$ M. D. Fraser, ${ }^{4}$ S. Holzinger, ${ }^{1}$ S. Brodbeck, ${ }^{1}$ M. Kamp, ${ }^{1}$ I. A. Shelykh,,${ }^{5,6}$ \\ C. Schneider, ${ }^{1}$ and S. Höfling ${ }^{1,7}$ \\ ${ }^{1}$ Technische Physik, Wilhelm-Conrad-Röntgen-Research Center for Complex Material Systems, Universität Würzburg, \\ Am Hubland, D-97074 Würzburg, Germany \\ ${ }^{2}$ QCD Labs, COMP Centre of Excellence, Department of Applied Physics and Low Temperature Laboratory (OVLL), \\ Aalto University, P.O. Box 13500, FI-00076 Aalto, Finland \\ ${ }^{3}$ National Research University of Information Technologies, Mechanics and Optics (ITMO), St. Petersburg 197101, Russia \\ ${ }^{4}$ Quantum Optics Research Group, RIKEN Center for Emergent Matter Science, 2-1 Hirosawa, Wako-shi, \\ Saitama 351-0198, Japan \\ ${ }^{5}$ Science Institute, University of Iceland, Dunhagi 3, IS-107 Reykjavik, Iceland \\ ${ }^{6}$ Division of Physics and Applied Physics, Nanyang Technological University, 637371 Singapore, Singapore \\ ${ }^{7}$ SUPA, School of Physics and Astronomy, University of St. Andrews, St. Andrews KYl6 9SS, United Kingdom
} (Received 12 April 2014; published 14 November 2014)

\begin{abstract}
In this work, we combine a systematic experimental investigation of the power- and temperaturedependent evolution of the spatial coherence function, $g^{(1)}(\boldsymbol{r})$, in a one dimensional exciton-polariton channel with a modern microscopic numerical theory based on a stochastic master equation approach. The spatial coherence function $g^{(1)}(\boldsymbol{r})$ is extracted via high-precision Michelson interferometry, which allows us to demonstrate that in the regime of nonresonant excitation, the dependence $g^{(1)}(\boldsymbol{r})$ reaches a saturation value with a plateau, which is determined by the intensity of the pump and effective temperature of the crystal lattice. The theory, which was extended to allow for treating incoherent excitation in a stochastic frame, matches the experimental data with good qualitative and quantitative agreement. This allows us to verify the prediction that the decay of the off-diagonal long-range order can be almost fully suppressed in one dimensional condensate systems.
\end{abstract}

DOI: 10.1103/PhysRevLett.113.203902

Introduction.-Exciton polaritons evolve in semiconductor microcavities as the result of strong coupling of optical and matter modes [1]. At sufficiently low concentrations (up to $\sim 10^{11} \mathrm{~cm}^{-2}$ ) they obey bosonic statistics, and owing to their small effective mass, which is about $10^{5}$ times smaller than the free electron mass, manifest quantum coherent properties at surprisingly high temperatures. Indeed, polariton condensation is observed at temperatures of tens of Kelvin in GaAs and CdTe-based structures [2,3] and up to room temperatures in wide band-gap materials [4-6]. Having lot of similarities with conventional BoseEinstein condensation (BEC) [1], polariton condensation reveals some important peculiarities. Differently from cold atoms, polaritons have a finite lifetime and in order to reach an equilibrium state their radiative decay should be compensated by a constant pumping of the system, which can be implemented either optically or electrically [7]. Moreover, the planar microcavity exciton-polariton system is inherently two dimensional (2D), and in accordance with the Hohenberg-Mermin-Wagner theorem the transition to $\mathrm{BEC}$ in a uniform system is only possible at zero temperature [8] for 2D as well as $1 \mathrm{D}$ geometries. Restriction of the system to a finite size, however, inhibits the excitation of density and phase fluctuations permitting the formation of a condensate or quasicondensate phase with a macroscopic coherence length $[9,10]$.
PACS numbers: 42.55.Sa, 78.20.Ls, 78.55.Cr, 78.67.De

There is a long-standing discussion in the literature addressing the following question: What should be the experimentally verifiable criterion of the polariton condensation? It is now commonly accepted that the onset of the off-diagonal long-range order (ODLRO), determined by the first-order spatial coherence function $g^{(1)}(\mathbf{r})$, can be considered as a smoking gun criterion, putting the latter function in the most intense focus of theoretical [11-14] and experimental $[2,15,16]$ research. In particular, polariton condensation in one dimensional channels has drawn special attention. It was demonstrated that polaritons trapped in a channel reveal the appearance of ODLRO in the condensation regime [16]. Moreover, the droplets of BECs in such systems can propagate over macroscopic distances, preserving their coherence properties and allowing for the efficient manipulation [17]. Recently, an experimental observation of room-temperature polariton condensation in a one dimensional $\mathrm{ZnO}$ channel has been reported [18]. These technological achievements open a route to the creation of polariton-based logic elements and optical integrated circuits working at relatively high temperatures [19]. Among the phases expected for a 1D polariton gas are the Tonks gas [20] and the condensate or quasicondensate phases [10], the former appearing when interactions come to dominate the energy scale and the condensate phases resulting for a sufficiently weakly 
interacting and finite-sized system. The quasicondensate, which still exhibits some phase fluctuations, is characterized by an exponentially decaying long-range order, but with an enhanced coherence length. Such a phase is typical for the weakly interacting dilute-gas atomic condensate. The low energy of phase fluctuations in this system prevents the formation of a true condensate phase with a plateauing long-range order, except at the lowest temperatures [10]. The decay of spatial coherence in a low-dimensional nonequilibrium (polariton) condensate has been studied through a mean-field treatment with a stochastic noise term [21] with the result that, provided the gas is interacting, similar behavior to that of equilibrium BEC is expected in 1D, 2D, and 3D systems. The results that we discuss in this Letter, however, distinctly place our 1D polariton condensate into the true BEC-like phase where both phase and density fluctuations are suppressed to the level that ODLRO can be observed with a BEC-like constant plateau in the spatial coherence at the largest distances.

On the theoretical side, for the description of the polariton dynamics in one dimension, an approach based on the Lindblad master equation technique has been developed [22]. Different from the approaches based on either Gross-Pitaevskii-type equations $[23,24]$ or semiclassical Boltzmann equations [25-27], it allows us to account for real space dynamics of the polariton droplets, processes of decoherence and energy relaxation provided by the interaction of polaritons with the thermal bath of acoustic phonons. Moreover, the calculation of two-point correlators necessary for the analysis of the transition between thermal and OLDRO phases became possible [13].

Here, we demonstrate that once the intensity of the pump reaches a threshold value, the system undergoes the transition from the thermal to the condensate phase accompanied by the onset of ODLRO. Theoretically, this transition is modeled using the original microscopic approach based on a stochastic density matrix formalism. We investigate the influence of temperature and pump intensity modulation on the coherence properties of the system and reveal good agreement between the theory and experiment.

Experiment.-The polariton channels [Fig. 1(a)] with a length of $200 \mu \mathrm{m}$ (width $5 \mu \mathrm{m}$ ) were etched into a high- $Q$ AlGaAs based $\lambda / 2$ microcavity with $12 \mathrm{GaAs}$ quantum wells and a $Q$ factor exceeding 10000 (see Supplemental Material [28]).

First, we investigate the power-dependent emission features of the microwire cavity via momentum resolved spectroscopy. The microwire is mounted in a helium flow cryostat and it is oriented parallel to the entrance slit of the spectrometer. Polaritons are injected with a nonresonant $\mathrm{cw}$ laser which is tuned to the energy of the first reflection minimum of the Bragg reflector. The Gaussian-shaped pumping spot has a diameter of $5 \mu \mathrm{m}$, and the laser beam is chopped with a duty cycle of 0.1 to reduce power-induced heating effects. As shown in Fig. 1(b), we observe a set of
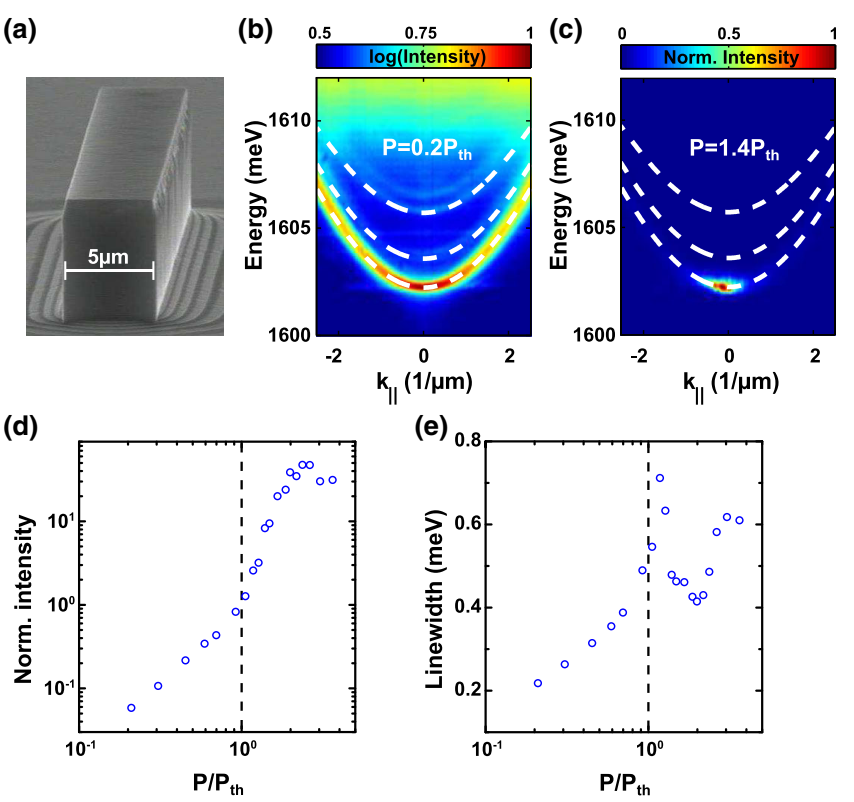

FIG. 1 (color online). (a) Scanning electron microscope image of a one dimensional polariton channel. (b) Power-dependent Fourier-space spectrum of the microwire emission below, and (c) above the regime of polariton condensation. (d) Input-output characteristics of the ground state emission from the wire. (e) Polariton linewidth as a function of the pump power.

parabolic dispersions which can be attributed to the ground state of the microwire as well as higher order lateral modes. Each dispersion is split into a mode doublet [34]. The energy-momentum detuning between the lowest photon mode $E_{C 1}\left(k_{\|}=0\right)$ and the exciton energy $E_{X}\left(k_{\|}=0\right)$ of the ground state amounts to $\delta=E_{C 1}\left(k_{\|}=0\right)-E_{X}\left(k_{\|}=0\right)=$ $-13 \mathrm{meV}$, which is less than the Rabi splitting $E_{\mathrm{RS}}=$ $14.5 \mathrm{meV}$. With the increase of the pump power, polaritons condense into the ground state which is manifested by its massive occupation [Fig. 1(c)]. The corresponding inputoutput characteristics of the power-dependent study is shown in Fig. 1(d). It features a distinct threshold which is typical for the onset of stimulated scattering leading to the growth of a condensate. Above $P=2.5 P_{t h}$ the intensity decreases again indicating excitation power-induced heating of the sample. At threshold, the linewidth of the polariton emission [shown in Fig. 1(e)] significantly reduces. This behavior is commonly assigned to an increased temporal coherence of the emission in the regime of polariton lasing [35]. Further, it increases again with the increase of the particle densities which can be attributed to dephasing resulted from the polariton-polariton interactions.

In order to investigate the spatial coherence properties of the polariton channels, we measure its spatial correlation function, $g^{(1)}\left(\mathbf{r}^{\prime}, \mathbf{r}^{\prime \prime}\right)$. We use a Michelson interferometer with a variable path length which overlaps the real space image of the polariton emission with its mirror image generated by a right angle prism. A schematic drawing of the optical setup is shown in Fig. 2(a) (it is similar to 


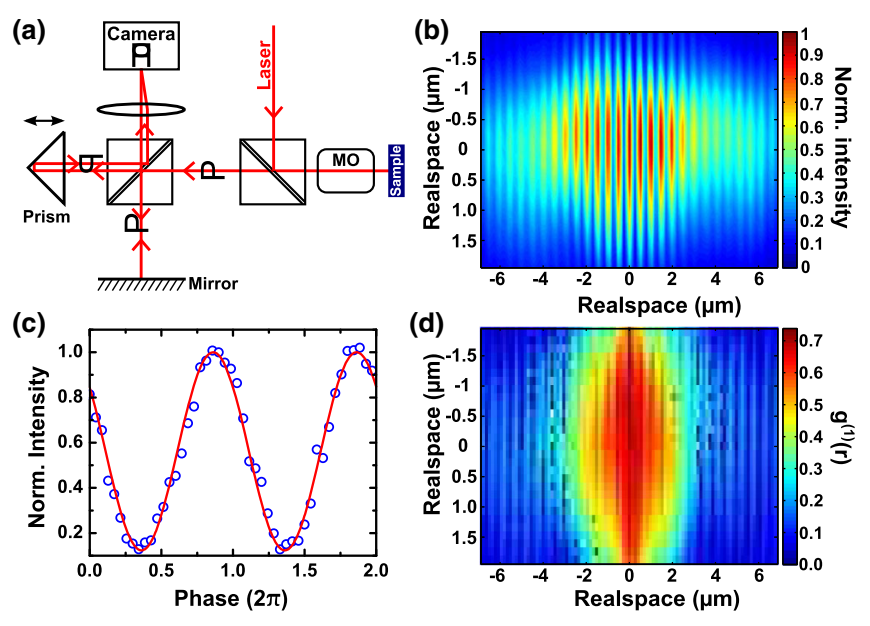

FIG. 2 (color online). (a) Schematic sketch of the Michelsoninterferometer setup for the determination of the spatial correlation function. (b) Typical interference pattern at an excitation power of $P=1.6 P_{t h}$. (c) By variation of the distance between the mirror and prism, the intensity of one pixel undergoes a sine function (blue circles). The red line shows the fitted sine from which the $g^{(1)}$ value and the phase can be calculated. (d) $g^{(1)}$ map received by fitting all the pixels of the camera image with a sine function above the condensation threshold at $P=1.6 P_{t h}$.

Ref. [36]). The overlapped real space images from the polariton wire are combined onto a CCD camera with high spatial resolution. The light from the excitation laser is filtered out with a long pass filter in the optical beam path. Figure 2(b) depicts the resulting interference pattern on the camera. By moving one arm of the interferometer, we can extract the interferograms. From the visibility of the interference fringes [see Fig. 2(c)], we can reconstruct the full spatial correlation function $g^{(1)}(r,-r)$ which is plotted in the color map in Fig. 2(d). It is interesting that, in contrast to the experimentally reported algebraic decay in 2D polariton systems $[15,36]$, the spatial coherence function of a one dimensional condensate is indicated to reach a constant plateau at large distances $[16,37,38]$. However, in these reports the clarity of this behavior is either obscured by large experimental errors resulting from the applied double slit technique [38] or by strong oscillations in the correlation function related to inhomogeneities of the 1D trap [16] and a comparably short long-range order in the range of $10 \mu \mathrm{m}$ [37]. For the purposes of assessing the long-range coherence behavior, the $1 \mathrm{D}$ polariton condensate in our experiments may roughly be characterized as a finite area but uniform 1D condensate due to its smooth steady-state profile. It is now well appreciated that the cw-pumped polariton condensate may be viewed as confined to an "effective trap" $[39,40]$ within which excitations over the entire condensate may be observed. Thus, despite ballistic expansion, the spatially extended steady-state profile represents the system size, and we may qualitatively apply the expected 1D correlation functions to this experimental system.
Theory.-Theoretically, the first-order spatial coherence can be determined as

$$
g^{(1)}\left(\mathbf{r}^{\prime}, \mathbf{r}^{\prime \prime}\right)=\frac{\chi\left(\mathbf{r}^{\prime}, \mathbf{r}^{\prime \prime}\right)}{\sqrt{\chi\left(\mathbf{r}^{\prime}, \mathbf{r}^{\prime}\right) \chi\left(\mathbf{r}^{\prime \prime}, \mathbf{r}^{\prime \prime}\right)}},
$$

where $\chi\left(\mathbf{r}^{\prime}, \mathbf{r}^{\prime \prime}\right)$ is the single-particle density matrix of the system in real space:

$$
\chi\left(\mathbf{r}^{\prime}, \mathbf{r}^{\prime \prime}\right)=\left\langle\hat{\Psi}^{\dagger}\left(\mathbf{r}^{\prime}\right) \hat{\Psi}\left(\mathbf{r}^{\prime \prime}\right)\right\rangle \equiv \operatorname{Tr}\left[\rho \hat{\Psi}^{\dagger}\left(\mathbf{r}^{\prime}\right) \hat{\Psi}\left(\mathbf{r}^{\prime \prime}\right)\right] .
$$

Here $\hat{\Psi}^{\dagger}(\mathbf{r}), \hat{\Psi}(\mathbf{r})$ are exciton-polariton field operators; $\rho$ is a full density matrix of the closed system (polariton system plus the environment).

In the case of a spatially homogeneous system, the firstorder coherence is only dependent on the relative distance: $g^{(1)}\left(\mathbf{r}^{\prime}, \mathbf{r}^{\prime \prime}\right)=g^{(1)}(r)$, where $r=\left|\mathbf{r}^{\prime}-\mathbf{r}^{\prime \prime}\right|$. In the ODLRO phase, the correlations do not decay even at very large distances, and thus $\lim _{r \rightarrow \infty} g^{(1)}(r) \neq 0$ [41]. In order to calculate $g^{(1)}(r)$ at different temperatures and pump intensities, we will employ a closed system of dynamic equations for the elements of the single-particle density matrix in the reciprocal space based on the Lindblad master equation techniques and account for the processes of polaritonpolariton and polariton-phonon interactions, pumping, and finite lifetime. The corresponding formalism was developed by us, and is described in greater detail elsewhere [22,42]. In those previous works, we treated the pulsed excitation of the condensate simply by introducing appropriate initial conditions [22], or we considered the $\mathrm{cw}$ resonant injection [42]. To accurately account for the effects of incoherent pumping, we had to extend our model by a stochastic approach introducing the random phase in the pumping term and performing statistical averaging. This modification represents a nontrivial and vitally important extension of our previous works to realistically model the experimental data. Upon finding the single-particle density matrix in the reciprocal space, the real space behavior can be found by the Fourier transform. The theoretical formalism is summarized in the Supplemental Material [28] and full details can be found in Refs. [13,22,42].

In the calculations, we used parameters (effective mass of polaritons, the Rabi splitting energy and detuning, the temperature for each set of data, and dimensions of the quantum wire) taken from the experimental data. The matrix element of polariton-polariton interaction was estimated using the expression $U \approx 3 E_{b} a_{B}^{2} / S$, where $E_{b}$ is the exciton binding energy, $a_{B}$ is its Bohr radius, and $S$ is the area of the wire, which gave $U \approx 4 \mathrm{neV}$. The polariton-polariton scattering rate was taken independent of the wave vector, for simplicity. The polariton-phonon scattering rate is calculated using Eq. (7) in [28] and the parameters there. The maximum value of the scattering rate reads $W \approx 38 \mathrm{neV}$. Pumping powers were chosen in accordance with the experimental data.

Results and discussion.-First, we investigate the behavior of coherence with the increase of the condensate density controlled by the intensity of the pump, $P$. Below the 

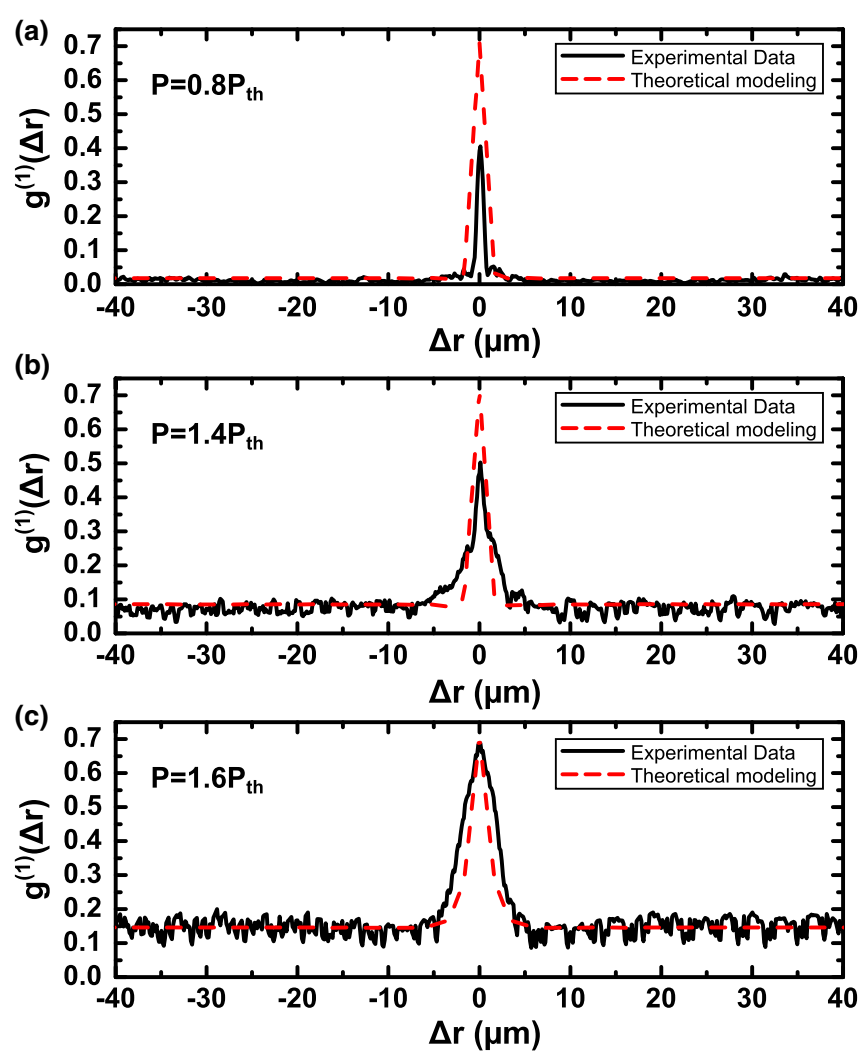

FIG. 3 (color online). $\quad g^{(1)}(\Delta r)$ for different excitation powers (a) below condensation threshold $P=0.8 P_{t h}$, (b) slightly above threshold $P=1.4 P_{t h}$ and (c) $P=1.6 P_{t h}$. With higher excitation powers the central peak width around $\Delta r=0$ increases and the value of the $g^{(1)}(\Delta r \rightarrow \infty)$ function reaches a plateau of about 0.15 at $P=1.6 P_{t h}$. The red dashed lines in (a)-(c) are the results of our theoretic modeling.

threshold of condensation, the coherence function $g^{(1)}(r)$ represents a resolution-limited sharp peak and a fast drop down to the noise level, which is shown in Fig. 3(a). After reaching the polariton lasing threshold $P_{t h}$ [Fig. 3(b)], this central peak starts to broaden, indicating the increase of the coherence [Fig. 3(c)]. In thermal equilibrium, the width of the peak is related to the thermal de Broglie wavelength $\lambda_{d B}$ of the Bose gas. Despite our system being clearly out of equilibrium, we follow this analogy and extract an effective de Broglie wavelength of $\lambda_{d B} \approx 8.5 \mu \mathrm{m}$ well above the condensation threshold, which is in good agreement with earlier reports [36]. More remarkable, the spatial correlation function acquires a nearly constant plateau in the regime of polariton condensation, which persists over the full range of the microwire significantly above the noise level.

For moderate pump powers (below two times the threshold power) we observe a consistent increase of the spatial coherence degree with the increase of polariton occupancy. This is exemplarily demonstrated in Fig. 3(c) for a pump power of $1.6 P_{t h}$. We can quantitatively reproduce this behavior with our theory, as shown in Figs. 3(a)-3(c): The spatial coherence is monotonically decaying over the distance $r$ and reaches some nonzero value $g_{\infty}^{(1)}$ for $r \rightarrow \infty$.
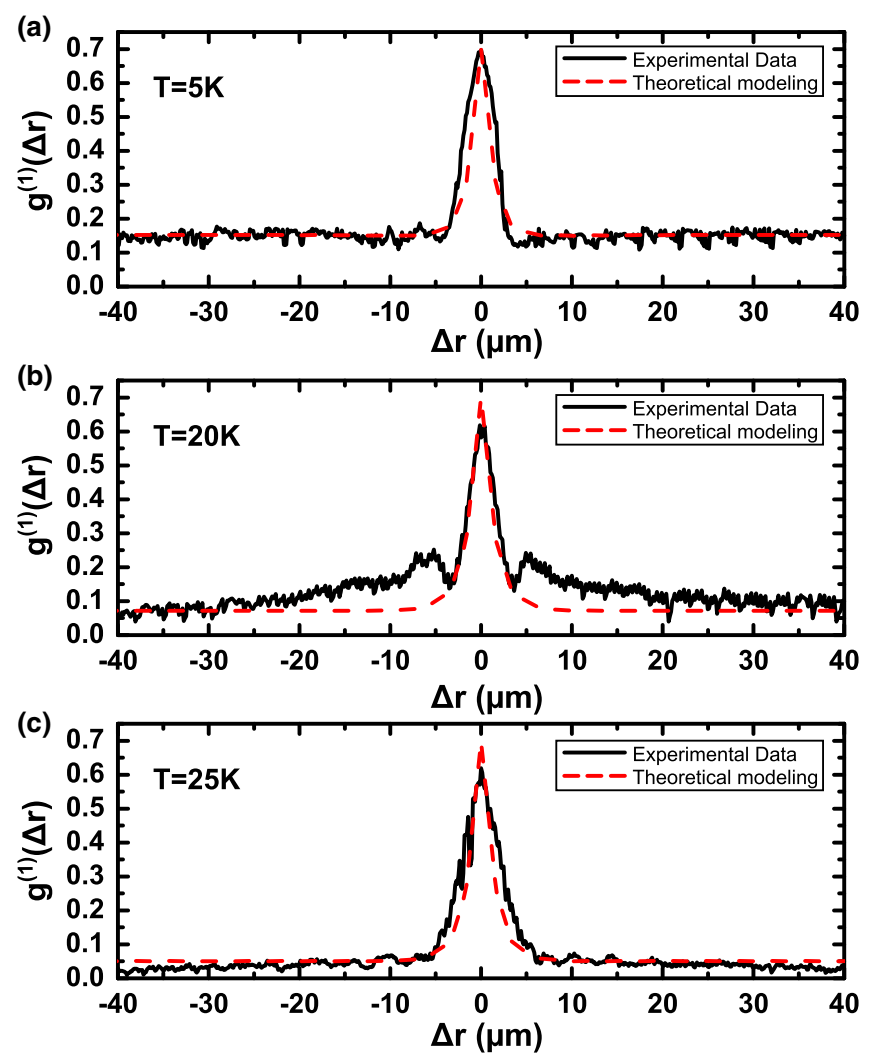

FIG. 4 (color online). $g^{(1)}(\Delta r)$ function for different sample temperatures (a) $T=5 \mathrm{~K}$, (b) $T=20 \mathrm{~K}$, and (c) $T=25 \mathrm{~K}$ at an excitation power of $P \approx 1.8 P_{t h}$. The red dashed lines are again the results of our model. With increasing temperatures, the plateau of $g^{(1)}(\Delta r \rightarrow \infty)$ disappears (c) $T=25 \mathrm{~K}$.

This value is determined by the percentage of the coherent fraction in the system and it increases with the pump power, $P$ (in case of absent strong localization effects). This behavior is also in good agreement with the theory.

Figure 4 serves as a manifestation of the thermal effects' influence on the long-range order. The figure depicts the evolution of $g^{(1)}(r,-r)$ as a function of temperature at a constant pump power of $1.8 P_{t h}$. The sample temperature is increased from 5 to $20 \mathrm{~K}$.

We observe a significant decrease of $g^{(1)}(r,-r)$ with an increase of the temperature, which strongly indicates the detrimental influence of acoustic phonons on the spatial coherence properties of the condensate. While for the highest temperature the correlation function drops to the noise level within the size of the pump spot in almost perfect agreement with theory [Fig. 4(c)], the occurrence of satellite peaks [Figs. 4(b), 3(b)] indicate some persisting influence of the sample disorder [37] or a boundary mode reflection. At a temperature of $T=25 \mathrm{~K}$ [Fig. 4(c)] the value of $g^{(1)}(r,-r)$ quenches to zero at distances $r>20 \mu \mathrm{m}$ and the transition from ODLRO to the thermal phase occurs.

Conclusion.-To summarize, we have investigated theoretically and experimentally spatial coherence properties of a one dimensional exciton-polariton microwire under different nonresonant pump intensities and temperatures. 
We have compared the experimental data with calculations, utilizing a stochastic master equation approach. It has been demonstrated that the $g^{(1)}(r)$ function has a plateau region which is determined by the intensity of nonresonant pumping and the effective temperature of the crystal lattice. This result places our condensate into the true BEC-like phase where both phase and density fluctuations are suppressed.

The authors would like to thank the State of Bavaria for financial support. I. A. S acknowledges the support of FP7 IRSES project POLAPHEN. I. G. S. was partially supported by the Academy of Finland through its Centre of Excellence Programs (Projects No. 250280 and No. 251748) and the Government of the Russian Federation, Grant No. 074-U01.

[1] A. Kavokin, J. Baumberg, G. Malpuech, and F. Laussy, Microcavities (Clarendon Press, Oxford, 2006).

[2] J.Kasprzak, M.Richard,S.Kundermann, A.Baas, P. Jeambrun, J. M. J. Keeling, F. M. Marchetti, M. H. Szymanska, R. André, J. L. Staehli, V. Savona, P. B. Littlewood, B. Deveaud, and L. S. Dang, Nature (London) 443, 409 (2006).

[3] R. Balili, V. Hartwell, D. Snoke, L. Pfeiffer, and K. West, Science 316, 1007 (2007).

[4] S. Christopoulos, G. B. H. von Högersthal, A. J. D. Grundy, P. G. Lagoudakis, A. V. Kavokin, J. J. Baumberg, G. Christmann, R. Butté, E. Feltin, J.-F. Carlin, and N. Grandjean, Phys. Rev. Lett. 98, 126405 (2007).

[5] T.-C. Lu, Y.-Y. Lai, Y.-P. Lan, S.-W. Huang, J.-R. Chen, Y.-C. Wu, W.-F. Hsieh, and H. Deng, Opt. Express 20, 5530 (2012).

[6] J. D. Plumhof, T. Stöferle, L. Mai, U. Scherf, and R. F. Mahrt, Nat. Mater. 13, 247 (2014).

[7] C. Schneider, A. Rahimi-Iman, N. Y. Kim, J. Fischer, I. G. Savenko, M. Amthor, M. Lermer, A. Wolf, L. Worschech, V. D. Kulakovskii, I. A. Shelykh, M. Kamp, S. Reitzenstein, A. Forchel, Y. Yamamoto, and S. Höfling, Nature (London) 497, 348 (2013).

[8] P. Hohenberg, Phys. Rev. 158, 383 (1967).

[9] D. S. Petrov, M. Holzmann, and G. V. Shlyapnikov, Phys. Rev. Lett. 84, 2551 (2000).

[10] D. S. Petrov, G. V. Shlyapnikov, and J. T. M. Walraven, Phys. Rev. Lett. 85, 3745 (2000).

[11] D. Sarchi and V. Savona, Phys. Rev. B 75, 115326 (2007).

[12] T. D. Doan, H. Thien Cao, D. B. Tran Thoai, and H. Haug, Phys. Rev. B 78, 205306 (2008).

[13] I. G. Savenko, I. A. Iorsh, M. A. Kaliteevski, and I. A. Shelykh, JETP Lett. 116, 32 (2013).

[14] V. N. Gladilin, K. Ji, and M. Wouters, Phys. Rev. A 90, 023615 (2014).

[15] H. Deng, G. S. Solomon, R. Hey, K. H. Ploog, and Y. Yamamoto, Phys. Rev. Lett. 99, 126403 (2007).

[16] F. Manni, K. G. Lagoudakis, B. Pietka, L. Fontanesi, M. Wouters, V. Savona, R. Andre, and B. Deveaud-Plédran, Phys. Rev. Lett. 106, 176401 (2011).

[17] G. Tosi, G. Christmann, N. G. Berloff, P. Tsotsis, T. Gao, Z. Hatzopoulos, P. G. Savvidis, and J. J. Baumberg, Nat. Phys. 8, 190 (2012).
[18] A. Trichet, L. Sun, G. Pavlovic, N. A. Gippius, G. Malpuech, W. Xie, Z. Chen, M. Richard, and L. S. Dang, Phys. Rev. B 83, 041302 (2011).

[19] T. C. H. Liew, A. V. Kavokin, T. Ostatnicky, M. Kaliteevski, I. A. Shelykh, and R. A. Abram, Phys. Rev. B 82, 033302 (2010).

[20] B. Paredes, A. Widera, V. Murg, O. Mandel, S. Fölling, I. Cirac, G. V. Shlyapnikov, T. W. Hänsch, and I. Bloch, Nature (London) 429, 277 (2004).

[21] A. Chiocchetta and I. Carusotto, Europhys. Lett. 102, 67007 (2013).

[22] I. G. Savenko, E. B. Magnusson, and I. A. Shelykh, Phys. Rev. B 83, 165316 (2011).

[23] I. Carusotto and C. Ciuti, Phys. Rev. Lett. 93, 166401 (2004).

[24] I. A. Shelykh, Y. G. Rubo, G. Malpuech, D. D. Solnyshkov, and A. Kavokin, Phys. Rev. Lett. 97, 066402 (2006).

[25] D. Porras, C. Ciuti, J. J. Baumberg, and C. Tejedor, Phys. Rev. B 66, 085304 (2002)

[26] T. D. Doan, H. T. Cao, D. B. Tran Thoai, and H. Haug, Phys. Rev. B 72, 085301 (2005).

[27] H. T. Cao, T. D. Doan, D. B. Tran Thoai, and H. Haug, Phys. Rev. B 77, 075320 (2008).

[28] See Supplemental Material at http://link.aps.org/ supplemental/10.1103/PhysRevLett.113.203902, which includes Refs. [29-33], for experimental details and the description of the theoretical formalism.

[29] H. Deng, G. Weihs, C. Santori, J. Bloch, and Y. Yamamoto, Science 298, 199 (2002).

[30] F. Tassone and Y. Yamamoto, Phys. Rev. B 59, 10830 (1999).

[31] C. Piermarocchi, F. Tassone, V. Savona, A. Quattropani, and P. Schwendimann, Phys. Rev. B 53, 15834 (1996).

[32] D. D. Solnyshkov, I. A. Shelykh, N. A. Gippius, A. V. Kavokin, and G. Malpuech, Phys. Rev. B 77, 045314 (2008).

[33] H. Carmichael, Quantum Optics 1: Master Equations and Fokker-Planck Equations (Springer, New York, 2007).

[34] A. Kuther, M. Bayer, T. Gutbrod, A. Forchel, P. A. Knipp, T. L. Reinecke, and R. Werner, Phys. Rev. B 58, 15744 (1998).

[35] J.-S. Tempel, F. Veit, M. Aßmann, L. E. Kreilkamp, A. Rahimi-Iman, A. Löffler, S. Höfling, S. Reitzenstein, L. Worschech, A. Forchel, and M. Bayer, Phys. Rev. B 85, 075318 (2012).

[36] G. Roumpos, M. Lohse, W. H. Nitsche, J. Keeling, M. H. Szymańska, P. B. Littlewood, A. Löffler, S. Höfling, L. Worschech, A. Forchel, and Y. Yamamoto, Proc. Natl. Acad. Sci. U.S.A. 109, 6467 (2012).

[37] A. Trichet, E. Durupt, F. Médard, S. Datta, A. Minguzzi, and M. Richard, Phys. Rev. B 88, 121407(R) (2013).

[38] E. Wertz, L. Ferrier, D. D. Solnyshkov, R. Johne, D. Sanvitto, A. Lemaître, I. Sagnes, R. Grousson, A. V. Kavokin, P. Senellart, G. Malpuech, and J. Bloch, Nat. Phys. 6, 860 (2010).

[39] G. Roumpos, W. H. Nitsche, S. Höfling, A. Forchel, and Y. Yamamoto, Phys. Rev. Lett. 104, 126403 (2010).

[40] E. A. Ostrovskaya, J. Abdullaev, A. S. Desyatnikov, M. D. Fraser, and Y. S. Kivshar, Phys. Rev. A 86, 013636 (2012).

[41] A. J. Leggett, Quantum Liquids: Bose Condensation and Cooper Pairing in Condensed-Matter Systems, Oxford Graduate Texts (Oxford University Press, New York, 2006).

[42] E. B. Magnusson, I. G. Savenko, and I. A. Shelykh, Phys. Rev. B 84, 195308 (2011). 